\title{
An Improved Quasi-Dynamic Analytical Method to Predict Skidding in Roller Bearings under Conditions of Extremely Light Loads and Whirling
}

\author{
Junning Li1,2,* - Wei Chen ${ }^{2}$ - Libo Zhang ${ }^{2}$ - Taofeng Wang2 \\ ${ }^{1}$ Xi'an Technological University, School of Mechatronic Engineering, China \\ 2 Xi'an Jiaotong University, Key Laboratory of Education Ministry for Modern Design and Rotor-Bearing System, China
}

\begin{abstract}
Slip often occurs in high-speed and light-load roller bearings (HSLLRBs) when the frictional drive force is inadequate to overcome the drag forces between the rolling elements and the raceway. Formerly, skidding analysis of HSLLRBs considering bearing whirling based on a simplified method using the Dowson-Higginson empirical model, although the analytical results of the cage slip fraction show significant discrepancies with the experimental data, as for extremely light radial loads. One of the main reasons is the inaccuracy of the evaluation of oil drag forces using the empirical equations. In this study, the elastohydrodynamic lubrication (EHL) method was adopted to calculate the oil film thickness and pressure distribution of HSLLRBs, so as to obtain more accurate oil drag forces. The cage speed and cage slip fraction were obtained by combining the whirl orbits, drag forces, load, kinematic equations and other related equations and then solved using the Newton-Raphson method. The skidding mechanism was investigated in terms of various operating parameters such as whirl orbit radii, radial load and viscosity. The results showed that the cage slip fraction and cage speed oscillate over time because of the whirl, which leads to an increase in the risk of bearing skidding damage. Under the extremely light load and high speed, the slip and influence of the whirl on bearing skidding increases as the whirl radius and radial load increases, while the viscosity shows a reverse trend. Therefore, in order to reduce slip and skidding damage, the whirl radius and radial load should be decreased suitably, while the viscosity should be increased moderately. A comparison between the calculated and experimental results shows that the proposed method is both feasible and valid.
\end{abstract}

Keywords: skid, whirl, roller bearing, squeeze film damper, EHL, operating parameters

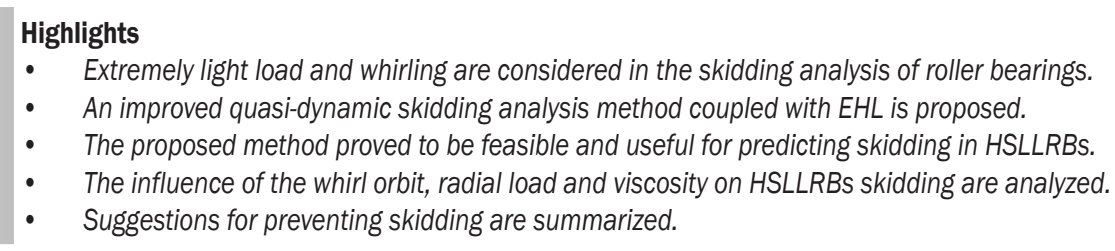

\section{INTRODUCTION}

Rolling-element bearings are key precision components used for rotor support in nearly all machinery. In high-speed and light-load roller bearings (HSLLRBs), such as the typical main shaft bearings of an aircraft engine, the centrifugal forces on the rollers are considered to play a major role in its mechanics. Under these conditions, the tractive forces between the inner raceway and the rollers are frequently insufficient to overcome the drag on the rolling element assembly, which results in the phenomenon of slip [1]. Extreme slip between the roller and the raceway can cause wear on the rolling contact surfaces and subsequently result in a smearing type of surface damage [2]. Several analytical models have been developed for the prediction of slip in roller bearings under different conditions [1] to [7]. Dowson and Higginson analyzed the effects of film thickness and frictional forces on cage slip and derived equations for calculating the various forces acting on a roller under rigid and elastohydrodynamic lubrication (EHL) regimes [3], but did not include the effect of centrifugal forces. Harris [4] and Harris and Kotzalas [5] proposed an analytical method for predicting skid in high-speed roller bearings; this method yielded good results under conditions of medium and excess loading. However, analytical data on the cage speed show significant discrepancies with the experimental data at extremely light radial loads. Poplawski developed a roller bearing model to estimate the cage slip and cage forces, whose analytical predictions of slip correlated well with his test results [6], however they were not in agreement with Harris's cage speed predictions and experimental results for extremely light radial loads. Since the slip velocities at the rolling element-cage contact are typically large, a constant friction coefficient is used at the rolling element-cage pocket contact [7].

Tu et al. [8] and Shao et al. [9] presented an analytical model to investigate skidding during acceleration of a rolling element bearing, which 
takes into consideration the contact force and friction force between the rolling elements races and the cage, as well as the gravity and centrifugal force of the rolling elements, and analyzed the effects of a localized surface defect on the vibration response of the cylindrical roller bearing. In addition, based on the computed tomography, Zbrowski and Matecki analyzed the grinding smudges and subsurface defects in roller bearing rings [10]. Zhang [11] and $\mathrm{Li}$ et al. [12] analyzed various factors that influence slip and developed skidding analysis software for high-speed roller bearings.

Most of the above mentioned theoretical analyses assume that roller bearings are installed directly in the bearing house. In practice, squeeze-film damper bearings (SFDBs) are widely used to inhibit the effects of vibration in a rotor-bearing system [13] and [14]. The inner ring of SFDBs is often mounted on the outer ring of the roller bearing with an interference fit that makes all of them whirl together, and thus inevitably influencing skidding damage of a roller bearing. Severe skidding of HSLLRBs in a whirling squeeze film damper often leads to vibration and failure of rotor-bearing systems or even entire machines.

Many studies have focused on HSLLRBs skidding analysis without considering bearing whirl. Based on a simplified method using the DowsonHigginson empirical model, $\mathrm{Li}$ and Chen analyzed the effects of different structure parameters on skidding of high-speed roller bearings in SFDBs considering bearing whirling [12], in which the radial load exceeds $500 \mathrm{~N}$. As for the extremely light radial loads, the analytical data on slip have significant discrepancies with the experimental data. One of the main reasons is the inaccuracy of the evaluation of oil drag forces with the Dowson-Higginson empirical equations, which have a significant effect on the bearing skidding analysis, especially under conditions of extremely light radial loads.

In this manuscript, HSLLRBs in a whirling squeeze film damper were taken as an example for skidding analysis. In order to obtain more accurate oil drag forces to assist in the skidding analysis of HSLLRBs, the EHL equations were solved using multigrid methods to obtain the values of the oil film thickness and pressure distribution, and then to acquire the fluid frictional drag forces. The skidding mechanism was investigated systematically in terms of various operating parameters such as whirl orbit radii, radial load and lubricating oil viscosity.

\section{SQUEEZE-FILM DAMPER BEARINGS}

Squeeze-film damper bearings (SFDBs) are widely used to inhibit the effects of vibration in a rotorbearing system. It has been shown that correctly designed SFDBs are a very effective means for reducing both the amplitude of rotor motion and the force transmitted to the bearing support [13] and [14]. In general, the rotating shaft carries a roller bearing, whose outer ring whirls with the inner ring of the SFDBs in the oil-filled clearance space between the inner and outer rings of the SFDBs. The outer ring of the bearing forms a damper so that rotation is inhibited by an anti-rotation pin [15] and [16]. Therefore, the damping effect of SFDBs mainly relies on an inner ring whirl, which squeezes the oil film in the clearance and forms resistance from the film. The inner ring of SFDBs is often mounted on the outer ring of roller bearing with an interference fit that makes all of them whirl together, thus inevitably influencing skidding damage to the roller bearing.

The HSLLRBs of a whirling squeeze film damper is taken as an example for skidding analysis. The contact between roller and raceway is viewed as a rigid contact, the roller purely rolls along the outer ring raceway for the unloaded zone, and the cage normal forces are equal for every roller over all contact areas of the roller bearing.

\section{MATHEMATICAL MODELS}

\subsection{Whirl Orbits}

Bearing whirl is majorly stimulated by shaft rotation. Therefore, the whirling frequency is relevant to shaft speed. In this manuscript, the authors considered the example of a roller and assumed its whirl orbits to be a circle with radius $e$ with simple harmonic vibration in the $x$ and $y$ directions [17] and [18].

Taking the time of the maximum amplitude as the initial moment, the coordinates of orbit center $O_{w}$ can be expressed as follows:

So,

$$
\begin{aligned}
& e_{x}=e \cos \omega_{w} t \\
& e_{y}=e \sin \omega_{w} t
\end{aligned}
$$

$$
\begin{aligned}
& \ddot{e}_{x}=-e \omega^{2} \cos \omega_{w} t, \\
& \ddot{e}_{y}=-e \omega^{2} \sin \omega_{w} t .
\end{aligned}
$$




\subsection{Fluid Frictional Drag Forces}

As for the extremely light radial loads, the cage slip shows significant discrepancies with the experimental results. One of the main reasons is the inaccuracy of the evaluation of oil drag forces using the DowsonHigginson empirical equations, which have a significant effect on the bearing skidding analysis, especially under conditions of extremely light radial loads [19]. The oil drag forces relate to the film thickness and pressure. The fluid frictional drag forces model of the HSLLRBs is shown in Fig. 1.

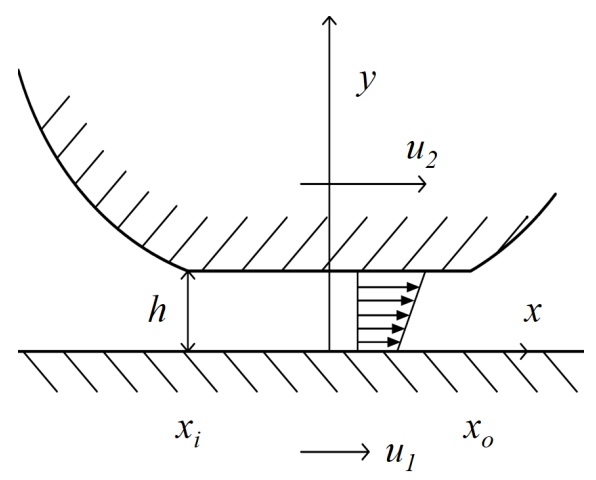

Fig. 1. Model of the fluid frictional drag forces

The fluid frictional drag forces $T$ can be expressed as follows [20]:

$$
T=-\int_{x_{i}}^{x_{o}} \frac{h}{2} \frac{\partial p}{\partial x} d x+\left(u_{2}-u_{1}\right) \int_{x_{i}}^{x_{o}} \frac{\eta}{h} d x .
$$

Here, $x_{i}$ and $x_{o}$ are the coordinates of the inlet and outlet.

Eq. (5) can be expressed in terms of dimensionless quantities as follows:

$$
\begin{aligned}
& \bar{F}_{i}=-\int_{X_{i}}^{X_{o}} \frac{H_{i} b^{3}}{8 l e R_{i}^{3}} \frac{\partial P}{\partial X} d X+\int_{X_{i}}^{X_{o}} \frac{\bar{V}_{i} R_{i} \bar{\eta}}{H_{i} l e b} d X, \\
& \bar{F}_{o}=-\int_{X_{i}}^{X_{o}} \frac{H_{o} b^{3}}{8 l e R_{o}^{3}} \frac{\partial P}{\partial X} d X+\int_{X_{i}}^{X_{o}} \frac{\bar{V}_{o} R_{o} \bar{\eta}}{H_{o} l e b} d X .
\end{aligned}
$$

The solution to the fluid frictional drag forces equations are related to the oil film thickness and pressure distribution, which are the main challenge and the emphasis of this study. Here, the EHL method is adopted to calculate the oil film thickness and pressure distribution of the roller bearing, and then the fluid frictional drag forces can be obtained using Eq. (6) to replace the inaccuracy values obtained using the Dowson-Higginson empirical equations.

\subsection{EHL Formulas}

As for the isothermal EHL in the line contact, the basic equations and their dimensionless forms can be expressed as follows:

Reynolds equation:

$$
\frac{d}{d X}\left(\varepsilon \frac{d P}{d X}\right)-\frac{d(\bar{\rho} H)}{d X}=0,
$$

where

$$
\varepsilon=\frac{\bar{\rho} H^{3}}{\bar{\eta} \zeta}, \quad \zeta=\frac{3 \pi^{2} \bar{U}}{4 W^{2}}
$$

The Reynolds boundary conditions of the equation are: in the area of the oil inlet $X=X_{\text {in }}, P=0$; and in the area of oil outlet $X=X_{\text {out }}, P=d P / d X=0$.

(1) Film thickness equation:

$$
H=H_{0}+\frac{X^{2}}{2}-\frac{1}{2 \pi} \int_{X_{\text {in }}}^{X_{\text {out }}} P(S) \ln (X-S)^{2} d S .
$$

(2) Viscosity equation varying with pressure (Roelands equation):

$$
\bar{\eta}=\exp \left\{\left(\ln \eta_{0}+9.67\right)\left[\left(1+5.1 \times 10^{-9} p_{H} P\right)^{z}-1\right]\right\}
$$

(3) Density equation:

$$
\bar{\rho}=\left(1+\frac{0.6 \times 10^{-9} p_{H} P}{1+1.7 \times 10^{-9} p_{H} P}\right) .
$$

(4) Load balancing condition equation:

$$
\int_{X_{\text {in }}}^{X_{\text {out }}} P d X=\frac{\pi}{2}
$$

Eqs. (7) to (11) can be expressed in terms of dimensionless quantities as follows: $X=\frac{x}{b}, \bar{\rho}=\frac{\rho}{\rho_{0}}$, $H=\frac{h R}{b^{2}}, \quad \bar{\eta}=\frac{\eta}{\eta_{0}}, \quad P=\frac{p}{p_{H}}, \quad \bar{U}=\frac{\eta_{0} u}{E^{\prime} R}, \quad W=\frac{w}{E^{\prime} R}$, $\frac{R}{b}=\sqrt{\frac{\pi}{8 W}}, \quad p_{H}=\frac{E^{\prime} b}{4 R}=\frac{E^{\prime}}{4} \sqrt{\frac{8 W}{\pi}}$.

Here, the EHL equations are solved using multigrid methods to obtain the values of the oil film thickness and pressure distribution, and then to acquire the fluid frictional drag forces using Eq. (6) to assist in the skidding analysis [19] and [20].

\subsection{Kinematics and Mechanical Models of the Roller}

The roller whirls along with the bearing. The kinematics model of a roller is shown in Fig. 2, and 
the forces acting on the roller at angular location $\varphi \mathrm{j}$ are shown in Fig. 3 [12].

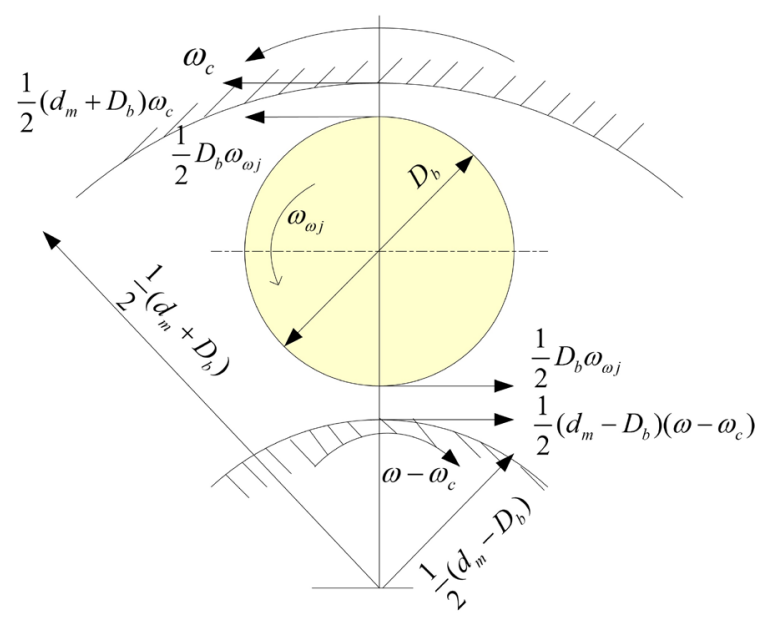

Fig. 2. Kinematics model

Consider a separate roller as an analysis object; its kinematics model is shown in Fig. 2. Thus, the sliding velocities can be expressed as follows:

$$
\begin{gathered}
V_{i j}=\frac{1}{2}\left(d_{m}-D_{b}\right)\left(\omega-\omega_{c}\right)-\frac{1}{2} D_{b} \omega_{\omega j}, \\
V_{o j}=\frac{1}{2}\left(d_{m}+D_{b}\right) \omega_{c}-\frac{1}{2} D_{b} \omega_{\omega j} .
\end{gathered}
$$

Additionally, the fluid entrainment velocities are defined as follows:

$$
U_{i j}=\frac{1}{2}\left(d_{m}-D_{b}\right)\left(\omega-\omega_{c}\right)+\frac{1}{2} D_{b} \omega_{\omega j},
$$

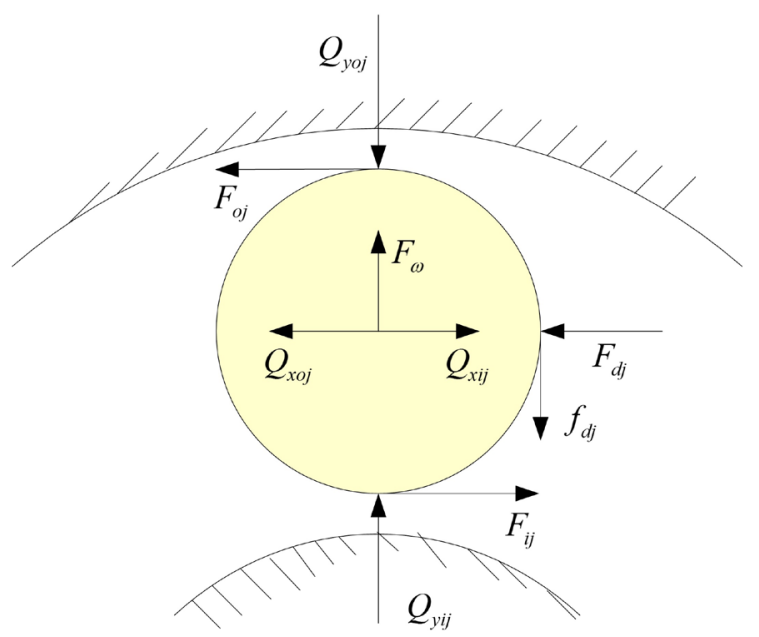

a)

$$
U_{o j}=\frac{1}{2}\left(d_{m}+D_{b}\right) \omega_{c}+\frac{1}{2} D_{b} \omega_{\omega j} .
$$

The sliding and the fluid entrainment velocities can be expressed in terms of dimensionless quantities as follows:

$$
\begin{gathered}
\bar{V}_{i j}=\frac{V_{i j} \mu_{0}}{E^{\prime} R_{i}}, \\
\bar{V}_{o j}=\frac{V_{o j} \mu_{0}}{E^{\prime} R_{o}}, \\
\bar{U}_{i j}=\frac{U_{i j} \mu_{0}}{E^{\prime} R_{i}}, \\
\bar{U}_{o j}=\frac{U_{o j} \mu_{0}}{E^{\prime} R_{o}} .
\end{gathered}
$$

In the loaded zone, the dynamic balance equations are expressed as follows:

$$
\begin{gathered}
\left.Q_{y j}+F_{\omega}-Q_{y o j}\right)=m_{r} \ddot{e}_{y}, \\
F_{i j}+Q_{x i j}-Q_{x o j}-F_{o j}-F_{d j}=m_{r} \ddot{e}_{x}, \\
F_{i j}+F_{o j}-f_{d j}=0 .
\end{gathered}
$$

In the unloaded zone, the dynamic balance equations are expressed as follows:

$$
\begin{gathered}
F_{\omega}-Q_{y o j u}=m_{r} \ddot{e}_{y}, \\
F_{d j u}-Q_{x o j u}-F_{o j u}=m_{r} \ddot{e}_{x}, \\
F_{o j u}+f_{d j}=0 .
\end{gathered}
$$

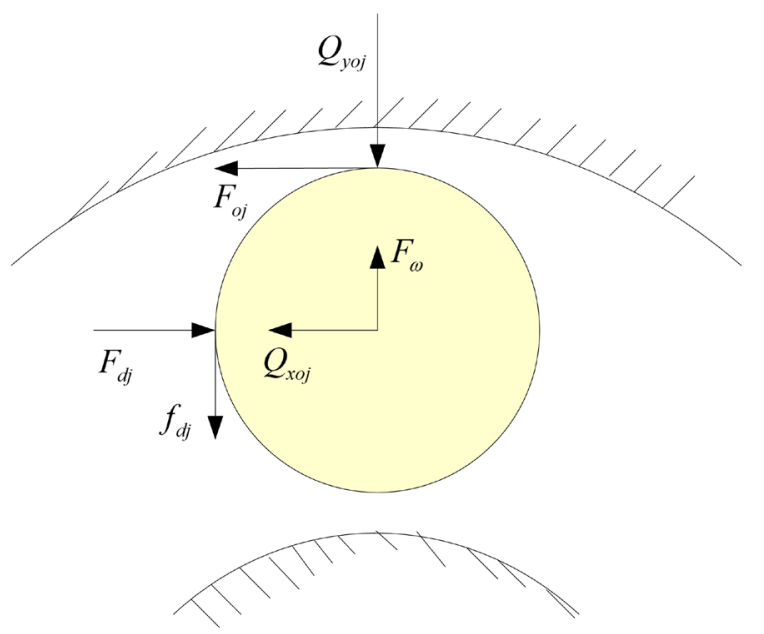

b)

Fig. 3. Mechanical models; a) loaded zone and b) unloaded zone 
Eq. (20) to (25) can be expressed in terms of dimensionless quantities.

In the loaded zone,

$$
\begin{gathered}
\bar{Q}_{y j j}+\left(\frac{R_{o}}{R_{i}}\right)\left(\bar{F}_{\omega}-\bar{Q}_{y o j}\right)=\frac{m_{r} \ddot{e}_{y}}{l E^{\prime} R_{o}}, \\
\bar{F}_{i j}+\bar{Q}_{x i j}-\left(\frac{R_{o}}{R_{i}}\right)\left(\bar{Q}_{x o j}+\bar{F}_{o j}+\bar{F}_{d j}\right)=\frac{m_{r} \ddot{e}_{x}}{l E^{\prime} R_{o}}, \\
\bar{F}_{i j}+\left(\frac{R_{o}}{R_{i}}\right) \bar{F}_{o j}-\bar{f}_{d j}=0 .
\end{gathered}
$$

In the unloaded zone,

$$
\begin{gathered}
\bar{F}_{\omega}-\bar{Q}_{y o j u}=\frac{m_{r} \ddot{e}_{y}}{l E^{\prime} R_{o}}, \\
\bar{F}_{d j u}-\bar{Q}_{x o j u}-\bar{F}_{o j u}=\frac{m_{r} \ddot{e}_{x}}{l E^{\prime} R_{o}}, \\
\bar{F}_{o j u}+\bar{f}_{d j}=0 .
\end{gathered}
$$

For entire rollers,

$$
\sum_{j=1}^{Z} \bar{F}_{d j}=0
$$

$Q_{y i j}$ and $Q_{y o j}$ denote normal forces transmitted by the raceways to the roller owing to bearing radial load and geometry. The new fluid frictional drag forces equations are adopted here:

$$
\begin{gathered}
\bar{F}_{i}=-\int_{X_{i}}^{X_{o}} \frac{H_{i} b^{3}}{8 l e R_{i}^{3}} \frac{\partial P}{\partial X} d X+\int_{X_{i}}^{X_{o}} \frac{\bar{V}_{i} R_{i} \bar{\eta}}{H_{i} l e b} d X, \\
\bar{F}_{o}=-\int_{X_{i}}^{X_{o}} \frac{H_{o} b^{3}}{8 l e R_{o}^{3}} \frac{\partial P}{\partial X} d X+\int_{X_{i}}^{X_{o}} \frac{\bar{V}_{o} R_{o} \bar{\eta}}{H_{o} l e b} d X, \\
\bar{Q}_{x i j}=18.4\left(1-\frac{D_{b}}{d_{m}}\right) G^{-0.3} \bar{U}_{i j}^{0.7},
\end{gathered}
$$

In addition, the cage drag normal force and tangential force acting on a roller are expressed as follows:

$$
\begin{aligned}
& F_{d j}=2 \int_{0}^{l_{e}} \frac{2.447 \eta / \omega_{j} \cdot\left(D_{b} x / 2\right)^{2}}{\sqrt{h_{0}}} d x, \\
& f_{d j}=2 \int_{0}^{l_{e}} \frac{0.5482 \eta / \omega_{j} \cdot\left(D_{b} x / 2\right)^{2}}{\sqrt{h_{0}}} d x .
\end{aligned}
$$

In this study, the EHL method is adopted to calculate the oil film thickness and pressure distribution of the roller bearing, which allows the fluid frictional drag forces to be determined. Eqs. (27) and (30) can be further substituted into Eq. (32), and then Eqs. (28), (31) and (32) form an equation set with $\mathrm{Z}+1$ equations. Then, the cage speed and cage slip fraction can be obtained by combining Eqs. (3) and (4) with the kinematic equations, load equations, Dowson-Higginson formulae, and other related equations and solved using the Newton-Raphson method [4] and [12].

\section{ANALYSIS AND DISCUSSION}

A single-row cylinder roller bearing is taken as an example. The default parameters are listed in Table 1. The effects of various operating parameters on the skidding of HSLLRBs taking into consideration bearing whirl are investigated.

Table 1. Details of the bearing and lubrication

\begin{tabular}{cccccc}
\hline Type & $d[\mathrm{~m}]$ & $d_{m}[\mathrm{~m}]$ & $G$ & $E^{\prime}[\mathrm{Pa}]$ & $\rho_{r}\left[\mathrm{~kg} / \mathrm{m}^{3}\right]$ \\
\hline NU214 & 0.07 & 0.0975 & 5000 & $2.28 \mathrm{e} 11$ & 7800 \\
\hline Cdyn & $d_{b}[\mathrm{~m}]$ & $l[\mathrm{~m}]$ & $Z$ & $P_{d}[\mathrm{~m}]$ & $\eta_{0}[\mathrm{P} \cdot \mathrm{s}]$ \\
\hline $1.37 \mathrm{e} 5$ & 0.013 & 0.013 & 18 & $2.5 \mathrm{e}-5$ & 0.08 \\
\hline
\end{tabular}

The cage slip fraction $S_{f}$ can be expressed as follows:

$$
\begin{gathered}
s_{f}=1-\frac{n}{n_{m}}, \\
n_{m}=\frac{1}{2} n_{i}\left(1-\frac{D_{b}}{d_{m}}\right) .
\end{gathered}
$$

\subsection{Effect of Different Whirl Radii on Skidding}

Bearing whirl induces the generation of additional forces in roller-cage and roller-rings pairs. More importantly, the oil film forces magnitudes of the roller-rings and roller-cage to oscillate as well. These forces oscillate over time, which often effect fatigue life and skidding damage to the bearing. Fig. 4 shows that the cage slip fraction and cage speed vary with time as the result of the whirl. In addition, the amplitudes of the cage speed and cage slip fraction increase with an increase in the whirl radius. So the degree of skidding can be reduced by suitably decreasing the whirl radius.

\subsection{Effect of Different Radial Loads on Skidding}

In contrast to the previous results, Fig. 5 shows that the cage slip fraction increases as the radial load increases, but that the cage speed shows a reverse trend. On the other hand, Table 2 shows that the amplitudes of cage speed and cage slip fraction decrease with an increase in the radial load. In other words, the influence of whirl on bearing skidding increases as the radial load increases. Therefore, the degree of skidding can be 

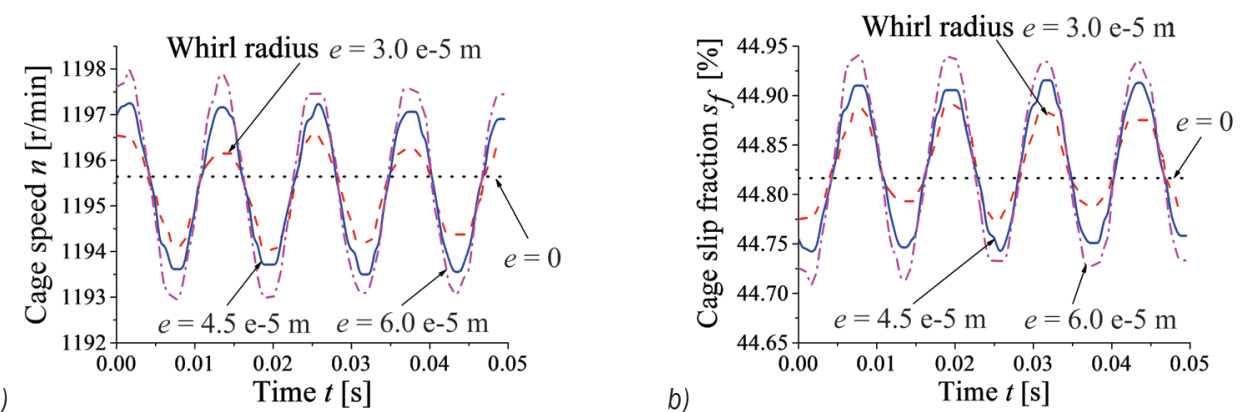

Fig. 4. Skidding analysis under different whirl radii ( $\mathrm{ni}=5000 \mathrm{r} / \mathrm{min} ; \mathrm{Fr}=200 \mathrm{~N} ; \mathrm{Cdyn} / \mathrm{P}=675) ;$ a) cage speed vs. whirl radius, and b) cage slip fraction vs. whirl radius
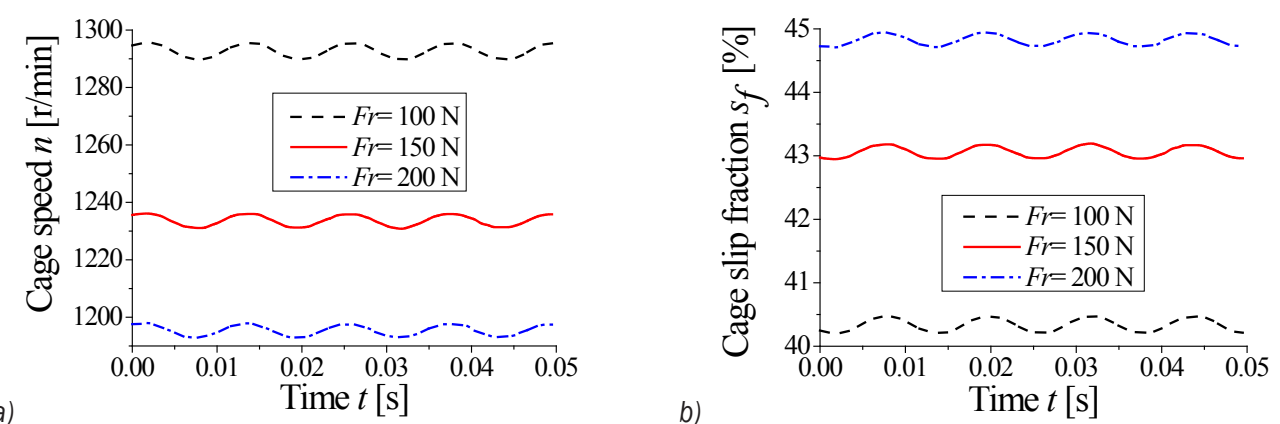

Fig. 5. Skidding analysis under different radial loads ( $\mathrm{ni}=5000 \mathrm{r} / \mathrm{min} ; \mathrm{e}=6.0 \mathrm{e}-5 \mathrm{~m}$ ); a) cage speed vs. radial load, and b) cage slip fraction vs. radial load
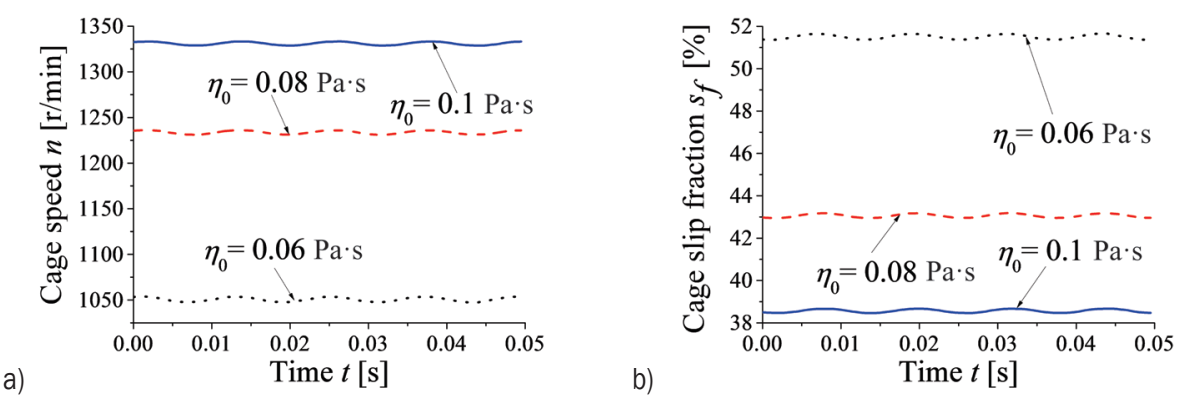

Fig. 6. Skidding analysis for different viscosities of lubricating oil ( $\mathrm{ni}=5000 \mathrm{r} / \mathrm{min} ; \mathrm{Fr}=150 \mathrm{~N} ; \mathrm{e}=6.0 \mathrm{e}-5 \mathrm{~m}$ ); a) cage speed vs. viscosity, and b) cage slip fraction vs. viscosity

reduced by suitably decreasing the radial load for extremely light radial loads conditions.

\subsection{Effect of Different Lubricating Oil Viscosities on Skidding}

In contrast to previous results, Fig. 6 shows that the cage slip fraction decreases as the viscosity increases, but that the cage speed shows a reverse trend. On the other hand, Table 3 shows that the amplitudes of cage speed and cage slip fraction decrease as the viscosity increases. In other words, whirl has a smaller influence on bearing skid as viscosity increases. That is, the degree of skidding can be reduced by suitably increasing the viscosity for extremely light radial load conditions.

\subsection{Discussion}

References [4] and [21] show that the experimental data on slip initially decreases and then increases as the radial load increases, which shows significant discrepancies with the analytical data for extremely light radial loads. One of the main reasons is the inaccuracy of the evaluation of the oil drag forces using the Dowson-Higginson empirical equations, 
Table 2. Results of fluctuate analysis data on various radial loads

\begin{tabular}{|c|c|c|c|c|c|c|}
\hline \multirow{2}{*}{ Value name } & \multicolumn{3}{|c|}{$n$} & \multicolumn{3}{|c|}{$S_{f}[\%]$} \\
\hline & $(F r=100 \mathrm{~N})$ & $(F r=150 \mathrm{~N})$ & $(F r=200 \mathrm{~N})$ & $(F r=100 \mathrm{~N})$ & $(F r=150 \mathrm{~N})$ & $(F r=200 \mathrm{~N})$ \\
\hline Minimum & 1289.81 & 1230.79 & 1192.92 & 40.21 & 42.95 & 44.71 \\
\hline Maximum & 1295.52 & 1236.13 & 1197.99 & 40.47 & 43.19 & 44.94 \\
\hline Mean & 1292.78 & 1233.70 & 1195.46 & 40.33 & 43.06 & 44.83 \\
\hline Fluctuation & 5.71 & 5.34 & 5.07 & 0.26 & 0.25 & 0.23 \\
\hline
\end{tabular}

Table 3. Results of fluctuate analysis data on various viscosities

\begin{tabular}{|c|c|c|c|c|c|c|}
\hline \multirow{2}{*}{ Value name } & \multicolumn{3}{|c|}{$n$} & \multicolumn{3}{|c|}{$S_{f}[\%]$} \\
\hline & $\left(\eta_{0}=0.06 \mathrm{~Pa} \cdot \mathrm{s}\right)$ & $\left(\eta_{0}=0.08 \mathrm{~Pa} \cdot \mathrm{s}\right)$ & $\left(\eta_{0}=0.1 \mathrm{~Pa} \cdot \mathrm{s}\right)$ & $\left(\eta_{0}=0.06 \mathrm{~Pa} \cdot \mathrm{s}\right)$ & $\left(\eta_{0}=0.08 \mathrm{~Pa} \cdot \mathrm{s}\right)$ & $\left(\eta_{0}=0.1 \mathrm{~Pa} \cdot \mathrm{s}\right)$ \\
\hline Minimum & 1047.45 & 1230.79 & 1328.81 & 51.37 & 42.95 & 38.46 \\
\hline Maximum & 1053.75 & 1236.13 & 1333.30 & 51.66 & 43.19 & 38.67 \\
\hline Mean & 1050.79 & 1233.70 & 1331.10 & 51.50 & 43.06 & 38.56 \\
\hline Fluctuation & 6.30 & 5.34 & 4.48 & 0.29 & 0.25 & 0.21 \\
\hline
\end{tabular}

which subsequently has a significant effect on the bearing skidding analysis, especially under conditions of extremely light radial loads. In this manuscript, a modified skidding analysis method is proposed to solve this issue. In order to verify the validity of this method, the analytical data are compared with the experimental data from the reference [21] without regard to the whirling motion, as shown in Fig. 7. Here, a roller bearing with 18 rollers is taken as an example with a diametral clearance of 2.5 e- $5 \mathrm{~m}$ and other parameters the same as those in Table 1. Fig. 7 shows that the shape of the analytical data curves is closely approximated by the experimental data under conditions of extremely light radial loads, where the critical value of the radial loads is $200 \mathrm{~N}$. The results show that the analytical method proposed in this manuscript is valid and feasible.

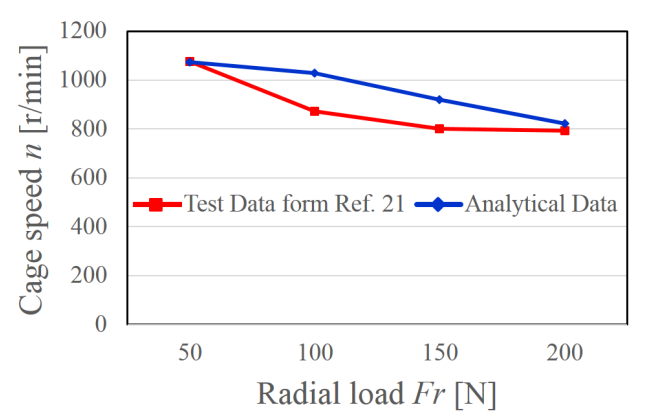

Fig. 7. Cage speed vs. radial load

$\left(n_{i}=4000 \mathrm{r} / \mathrm{min} ; Z=16 ; P_{d}=2.5 \mathrm{e}-5 \mathrm{~m}\right)$

HSLLRBs in a whirling squeeze film damper are taken as an example. The EHL equations were solved using multigrid methods to obtain the values of the oil film thickness and pressure distribution in order to determine the fluid frictional drag forces and then the skidding mechanism was systematically investigated. The results showed that the cage slip fraction and cage speed oscillate over time because of the whirl, which leads to an increase in the risk of bearing skidding damage. Under the extremely light load and high speed, the slip and influence of the whirl on bearing skidding increases as the whirl radius and radial load increases, while the oil viscosity shows a reverse trend. Slip is often related to skidding damage to the roller bearing, especially under conditions of high speed and light load. So, in order to reduce the risk of skidding damage, slip should be reduced first.

\section{CONCLUSIONS}

In this study, the EHL method is adopted to calculate the oil film thickness and pressure distribution of the roller bearing under extremely light load, then the oil drag forces are obtained to replace the inaccurate values obtained using the Dowson-Higginson empirical equations. Cage speed and cage slip fraction are then obtained by combining the whirl orbits, drag forces, load, kinematic equations and other related equations, which are then solved by the NewtonRaphson method. Finally, the skidding mechanism is investigated in terms of various operating parameters such as whirl orbit radii, radial load and lubricating oil viscosity.

(1) The cage slip fraction and cage speed oscillate over time because of the whirl, which leads to an increase in the risk of bearing skidding damage. The degree of skid varies directly with the whirl radius, so the degree of skidding can be reduced by suitably decreasing the whirl radius. 
(2) In contrast to the previous results, the cage slip fraction increases as the radial load increases under the extremely light load and high speed, but the cage speed shows a reverse trend. On the other hand, the influence of whirl on bearing skidding decreases as the radial load increases. Therefore, the degree of skidding can be reduced by suitably decreasing the radial load for extremely light radial loads conditions.

(3) In contrast to the previous results, the cage slip fraction decreases as the viscosity increases under the extremely light load and high speed, but the cage speed shows a reverse trend. On the other hand, whirl has a smaller influence on bearing skid as viscosity increases. That is, the degree of skidding can be reduced by suitably increasing the viscosity for extremely light radial loads conditions.

Therefore, in order to decrease the slip so as to reduce the risk of bearing skidding damage, the whirl radius and radial load should be decreased suitably, while the oil viscosity should be increased suitably. In this paper, the evaluation did not take in account all losses, such as, for example, churning losses. The authors will focus more on this valuable issue in the future.

\section{ACKNOWLEDGEMENT}

This research was supported by the National Natural Science Foundation of China under grant No. 59990470 and No. 51505361 and by the Fundamental Research Funds for Central Universities.

\section{NOMENCLATURE}

$n_{i} \quad$ bearing inner ring speed, $[\mathrm{r} / \mathrm{min}]$

$n \quad$ cage speed, [r/min]

$n_{m} \quad$ cage theoretical speed, [r/min]

$\omega_{n} \quad$ shaft angular velocity, $[\mathrm{rad} / \mathrm{s}]$

$\omega_{o}$ bearing outer ring angular velocity, $[\mathrm{rad} / \mathrm{s}]$

$\omega_{c}$ cage angular velocity, $[\mathrm{rad} / \mathrm{s}]$

$\omega_{\omega j}$ roller angular velocity about its own axis, [rad/s]

$S_{f} \quad$ cage slip fraction

$\varphi \quad$ roller angular location, [rad]

$D_{b}$ roller diameter, [m]

$l$ roller length, $[\mathrm{m}]$

$m_{r} \quad$ roller mass, $[\mathrm{kg}]$

$d$ bearing inner diameter, [m]

$D$ bearing outer diameter, $[\mathrm{m}]$

$d_{m} \quad$ bearing pitch diameter, $[\mathrm{m}]$

$P_{d} \quad$ diametral clearance, $[\mathrm{m}]$
$Z \quad$ number of rollers per row

$Z_{u} \quad$ number of unloaded rollers per row

$G \quad$ dimensionless material parameter

$E^{\prime} \quad$ equivalent elastic modulus, $[\mathrm{Pa}]$

$\rho_{r} \quad$ roller density, $\left[\mathrm{kg} / \mathrm{m}^{3}\right]$

$\rho$ lubricant density, $\left[\mathrm{kg} / \mathrm{m}^{3}\right]$

$\eta_{0} \quad$ absolute viscosity, [Pa.s]

$R$ radius of equivalent cylinder, [m]

$e \quad$ whirl circle radius, $[\mathrm{m}]$

$F \quad$ fluid frictional drag force, $[\mathrm{N}]$

$F_{r} \quad$ radial load, [N]

$F_{d} \quad$ cage drag normal force acting on a roller, [N]

$f_{\mathrm{dj}} \quad$ cage drag tangential force acting on a roller, [N]

$F_{\omega} \quad$ roller centrifugal force, $[\mathrm{N}]$

$Q_{x} \quad$ fluid force in direction of orbital motion, [N]

$Q_{y} \quad$ raceway-roller normal loading, [N]

$H$ dimensionless oil film thickness

$H_{0} \quad$ dimensionless center film thickness without load

$u$ velocity, $[\mathrm{m} / \mathrm{s}]$

$U$ entrainment velocity, $[\mathrm{m} / \mathrm{s}]$

$W$ bearing radial load per unit length, $[\mathrm{N} / \mathrm{m}]$

$\mathrm{S} \quad$ integral parameters

$P_{H} \quad$ maximum film pressure $[\mathrm{Pa}]$

Subscripts:

$i \quad$ inner raceway

$o$ outer raceway

j roller located at $\varphi_{j}$

$u$ unloaded roller

$c$ cage or orbital motion

\section{REFERENCES}

[1] Markho, P.H., Smith, B.V., Lalor, M.J. (1981). An advanced apparatus for the study of roller and cage slip in high-speed roller bearings. Journal of Tribology, vol. 103, no. 1, p. 46-54, DOl:10.1115/1.3251613.

[2] Tassone, B.A. (1979). Roller bearing slip and skidding damage. Journal of Aircraft, vol. 12, no. 4, p. 281-287, DOI:10.2514/3.44445.

[3] Dowson, D., Higginson, G. (1963). Theory of roller bearing lubrication and deformation. Proceedings of Convention on Lubrication and Wear, London, p. 216-227.

[4] Harris, T.A. (1966). An Analytical Method to Predict Skidding in High Speed Roller Bearings. ASLE Transactions, vol. 9, no. 3, p. 229-241, DOl:10.1080/05698196608972139.

[5] Harris, T.A., Kotzalas, M.N. (2006). Roller bearing Analysis: Advanced Concepts of Bearing Technology. Wiley, New York.

[6] Poplawski, J. (1972). Slip and Cage Forces in a High-Speed Roller Bearing. Journal of Lubrication Techology, vol. 94, no. 2, p. 143-150, Dol:10.1115/1.3451660.

[7] Ghaisas N., Wassgren C.R., Sadeghi, F. (2004). Cage instabilities in cylindrical roller bearing. Journal of Tribology, vol. 126, no. 4, p. 681-689, Dol:10.1115/1.1792674. 
[8] Tu W., Shao Y., Mechefske C.K. (2012). An analytical model to investigate skidding in rolling element bearings during acceleration. Journal of Mechanical Science and Technology, vol. 26, no. 8, p. 2451-2458, Dol:10.1007/s12206-012-0627-9.

[9] Shao, Y., Liu, J., Ye, J. (2014). A new method to model a localized surface defect in a cylindrical roller-bearing dynamic simulation. Proceedings of the Institution of Mechanical Engineers, Part J: Journal of Engineering Tribology, vol. 228, no. 2, p. 140-159, DOI:10.1177/1350650113499745.

[10] Zbrowski, A., Matecki, K. (2014). The use of computed tomography to analyse grinding smudges and subsurface defects in roller bearing rings. Strojniški vestnik - Journal of Mechanical Engineering, vol. 60, no. 11, p. 709-715, D0l:10.5545/sv-jme.2014.1817.

[11] Zhang L. (2009). Skidding Analysis of Cylindrical Roller Bearing. MSc. Thesis, Xi'an Jiaotong University, Xi'an. (in Chineese)

[12] Li, J., Chen, W. (2014). Effects of different structure parameters on skidding of high-speed roller bearing considering bearing whirling. Journal of Vibroengineering, vol. 16, no. 2, p. 748-760.

[13] Delgado A., Andres L.S. (2010). Identification of force coefficients in a squeeze film damper with a mechanical seal: large contact force. Journal of Tribology, vol. 132, no. 3, p. 032201-032207, DOI:10.1115/1.4001458.

[14] Shende, R.W., Sane, S.K. (2014). Squeeze film damping for aircraft gas turbines. Defence Science Journal, vol. 38, no. 4, p. 439-456, D0I:10.14429/dsj.38.5874.
[15] Pietra L.D. (2000). Analytical and experimental investigation of squeeze-film dampers executing circular orbits. Meccanica, vol. 35, no. 2, p. 133-157, D0l:10.1023/A:1004881316965.

[16] Pietra L.D., Adiletta G. (2002). The squeeze Film Damper over four decades of investigations. Part I: Characteristics and operating features. The Shock and Vibration Digest, vol. 34, no. 1, p. 3-26.

[17] Zhang, J., Roberts, J.B., Ellis, J. (1994). Experimental behavior of a short cylindrical squeeze film damper executing circular centered orbits. Journal of Tribology, vol. 116, no. 3, p. 528534, Dol:10.1115/1.2928876.

[18] Defaye, C., Arghir, M., Bonneau, O., Carpentier, P., Debailleux, C., Imbourg, F. (2006). Experimental study of the radial and tangential forces in a whirling squeeze film damper. Tribology transactions, vol. 49, no. 2, p. 271-278, DOl:10.1080/05698190600614916.

[19] Dowson, D., Higginson, G. (1966). Elasto-Hydrodynamic Lubrication: The Fundamentals of Roller and Gear Lubrication. Pergamon Press, Oxford.

[20] Wen, S., Yang, P. (1992). Elastohydrodynamic Lubrication. Tsinghua University Press, Beijing.

[21] Chen, G., Li, J., Xu, J., Li, D. (1996). Coupling Kinematics with EHL Theory in Analysis of High-Speed Roller Bearing. Journal of Northwestern Polytechnical University, vol. 14, no. 1, p. 91-94. 